\title{
Analytic Solutions of the Kadomtsev-Petviashvili Equation with Power Law Nonlinearity Using the Sine-Cosine Method
}

\author{
S. Arbabi Mohammad-Abadi \\ Department of Mathematics, Anar Branch, Islamic Azad University, Anar, Iran
}

\begin{abstract}
In this paper, a sine-cosine method is used to construct many periodic and solitary wave solutions to Kadomtsev-Petviashvili equation with power law nonlinearity. Many new families of exact traveling wave solutions of the Kadomtsev-Petviashvili equation with power law nonlinearity are successfully obtained.
\end{abstract}

Keywords Sine-Cosine Method, Kadomtsev-Petviashvili Equation, Periodic Solution

\section{Introduction}

Nonlinear partial differential equations (NPDEs) are widely used to describe complex phenomena in various fields of science, especially in physics. Therefore solving nonlinear problems plays an important role in nonlinear sciences. Many effective methods of obtaining explicit solutions of NPDEs have been presented such as the tanh-method[1-3],the extended tanh method[4-6], the sine-cosine method[7-10], the homogeneous balance method[11], homotopy analysis method[12-18], the F-expa nsion method[19],three-wave method[20-22], extended homoclinic test approach[23-25], the (G'/G)-expansion method[26] and the exp-function method[27-30].

In this paper, by means of the Sine-cosine method, we will obtain some analytic solutions for the KadomtsevPetviashvili equation with power law nonlinearity. In the following section we have a brief review on the Sine-cosine method and in Section 3 and 4 , we apply the Sine-cosine method to obtain analytic solutions of the KadomtsevPetviashvili equation with power law nonlinearity. Finally, the paper is concluded in Section 5.

\section{The Sine-cosine method}

1. We introduce the wave variable $\xi=x-c t$ into the PDE

$$
P\left(u, u_{t}, u_{x}, u_{t t}, u_{x x}, u_{t x}, \ldots\right)=0
$$

where $u(x, t)$ is traveling wave solution. This enables us

* Corresponding author:

arbabi_math87@yahoo.com (S. Arbabi Mohammad-Abadi)

Published online at http://journal.sapub.org/ajcam

Copyright (C) 2011 Scientific \& Academic Publishing. All Rights Reserved to use the following changes:

$$
\frac{\partial}{\partial t}=-c \frac{\partial}{\partial \xi}, \frac{\partial^{2}}{\partial t^{2}}=c^{2} \frac{\partial^{2}}{\partial \xi^{2}}, \frac{\partial}{\partial x}=\frac{\partial}{\partial \xi}, \frac{\partial^{2}}{\partial x^{2}}=\frac{\partial^{2}}{\partial \xi^{2}}, \ldots
$$

One can immediately reduce the nonlinear PDE (1) into a nonlinear ODE

$$
Q\left(u, u_{\xi}, u_{\xi \xi}, u_{\xi \xi \xi}, \ldots\right)=0 .
$$

The ordinary differential equation (3) is then integrated as long as all terms contain derivatives, where we neglect integration constants.

2. The solutions of many nonlinear equations can be expressed in the form[8]

$$
u(\xi)=\left\{\begin{array}{cc}
\lambda \sin ^{\beta}(\mu \xi), & |\xi| \leq \frac{\pi}{\mu}, \\
0 & \text { otherwise }
\end{array}\right.
$$

or in the form

$$
u(\xi)=\left\{\begin{array}{cc}
\lambda \cos ^{\beta}(\mu \xi), & |\xi| \leq \frac{\pi}{2 \mu}, \\
0 & \text { otherwise, }
\end{array}\right.
$$

where $\lambda, \mu$ and $\beta \neq 0$ are parameters that will be determined, $\mu$ and $c$ are the wave number and the wave speed respectively. We use

$$
\begin{aligned}
& u(\xi)=\lambda \sin ^{\beta}(\mu \xi), \\
& u^{n}(\xi)=\lambda^{n} \sin ^{n \beta}(\mu \xi), \\
& \left(u^{n}\right)_{\xi}=n \mu \beta \lambda^{n} \cos (\mu \xi) \sin ^{n \beta-1}(\mu \xi), \\
& \left(u^{n}\right)_{\xi \xi}=-n^{2} \mu^{2} \beta^{2} \lambda^{n} \sin ^{n \beta}(\mu \xi) \\
& \quad+n \mu^{2} \lambda^{n} \beta(n \beta-1) \sin ^{n \beta-2}(\mu \xi),
\end{aligned}
$$

and the derivatives of (5) becoms

$$
\begin{aligned}
& u(\xi)=\lambda \cos ^{\beta}(\mu \xi), \\
& u^{n}(\xi)=\lambda^{n} \cos ^{n \beta}(\mu \xi), \\
& \left(u^{n}\right)_{\xi}=-n \mu \beta \lambda^{n} \sin (\mu \xi) \cos ^{n \beta-1}(\mu \xi), \\
& \left(u^{n}\right)_{\xi \xi}=-n^{2} \mu^{2} \beta^{2} \lambda^{n} \cos ^{n \beta}(\mu \xi) \\
& \quad+n \mu^{2} \lambda^{n} \beta(n \beta-1) \cos ^{n \beta-2}(\mu \xi),
\end{aligned}
$$


and so on for other derivatives.

3. We substitute (6) or (7) into the reduced equation obtained above in (3), balance the terms of the cosine functions when (7) is used, or balance the terms of the sine functions when (6) is used, and solving the resulting system of algebraic equations by using the computerized symbolic calculations. We next collect all terms whit same power in $\cos ^{k}(\mu \xi)$ or $\sin ^{k}(\mu \xi)$ and set to zero their coefficients to get a system of algebraic equations among the unknowns $\mu, \beta$ and $\lambda$. We obtained all possible value of the parameters $\mu, \beta$ and $\lambda[7]$.

\section{The (1+2)-Dimensional KP Equation with Power Law Nonlinearity}

The dimensionless form of the $(1+2)$-dimensional KP equation, with power law nonlinearity, that is going to be studied in this paper is given by[31]

$$
\left(u_{t}+a u^{n} u_{x}+u_{x x x}\right)_{x}+b u_{y y}=0 .
$$

Here in Eq. (8), $a$ and $b$ are real valued constants. After that we use the transformation

$$
u(x, y, t)=\varphi(\xi), \quad \xi=x+y-c t
$$

where $c$ is constant. There for the Eq. (8) converts to

$$
\left(-c \varphi^{\prime}+a \varphi^{n} \varphi^{\prime}+\varphi^{\prime \prime \prime}\right)^{\prime}+b \varphi^{\prime \prime}=0
$$

where by integrating twice we obtain

$$
(b-c) \varphi+\frac{a}{n+1} \varphi^{n+1}+\varphi^{\prime \prime}=0,
$$

substituting (4) into (11) gives

$$
\begin{aligned}
& (b-c) \lambda \sin ^{\beta}(\mu \xi)+\frac{a}{n+1} \lambda^{(n+1)} \sin ^{(n+1) \beta}(\mu \xi) \\
& \left.-\mu^{2} \beta^{2} \lambda \sin ^{\beta}(\mu \xi)+\mu^{2} \lambda \beta(\beta-1) \sin ^{\beta-2}(\mu \xi)\right)=0
\end{aligned}
$$

Equating the exponents and the coefficients of each pair of the sine functions we find the following system of algebraic equations:

$$
\begin{aligned}
& (\beta-1) \neq 0, \\
& \beta-2=(n+1) \beta, \\
& (b-c) \lambda-\mu^{2} \beta^{2} \lambda=0, \\
& \lambda \mu^{2} \beta(\beta-1)+\frac{a}{n+1} \lambda^{(n+1)}=0
\end{aligned}
$$

Solving the system (13) yields

$$
\beta=-\frac{2}{n}, \mu=\frac{1}{2} \sqrt{b-c}, \lambda=\left[\frac{(n+2)(n+1)(c-b)}{2 a}\right]^{\frac{1}{n}}
$$

where $\mathrm{c}$ is a free parameter. Hence, for $b>c$, the following periodic solutions

$$
u(\xi)=\left\{\frac{(n+2)(n+1)(c-b)}{2 a} \csc ^{2}\left[\frac{\sqrt{b-c}}{2}(\xi)\right]\right\}^{\frac{1}{n}}
$$

where $0<\frac{\sqrt{b-c}}{2}(\xi)<\pi$, and

$$
u(\xi)=\left\{\frac{(n+2)(n+1)(c-b)}{2 a} \sec ^{2}\left[\frac{\sqrt{b-c}}{2}(\xi)\right]\right\}^{\frac{1}{n}}
$$

where $\left|\frac{\sqrt{b-c}}{2}(\xi)\right|<\frac{\pi}{2}$.

However, for $c>b$, the following periodic solutions

$$
\begin{aligned}
& u(\xi)=\left\{\frac{(n+2)(n+1)(b-c)}{2 a} \operatorname{csch}^{2}\left[\frac{\sqrt{c-b}}{2}(\xi)\right]\right\}^{\frac{1}{n}}, \\
& u(\xi)=\left\{\frac{(n+2)(n+1)(c-b)}{2 a} \operatorname{sech}^{2}\left[\frac{\sqrt{b-c}}{2}(\xi)\right]\right\}^{\frac{1}{n}} .
\end{aligned}
$$

\section{The (1+3)-Dimensional KP Equation with Power Law Nonlinearity}

The dimensionless form of the $(1+3)$-dimensional KP equation, with power law nonlinearity, that is going to be studied in this paper is given by[32]

$$
\left(u_{t}+a u^{n} u_{x}+u_{x x x}\right)_{x}+b u_{y y}+c u_{z z}=0
$$

Here in Eq. (17), $a, b$ and $c$ are real valued constants. After that we use the transformation

$$
u(x, y, t)=\varphi(\xi), \quad \xi=x+y+z-m t
$$

where $m$ is constant. There for the Eq. (17) converts to

$$
\left(-m \varphi^{\prime}+a \varphi^{n} \varphi^{\prime}+\varphi^{\prime \prime \prime}\right)^{\prime}+(b+c) \varphi^{\prime \prime}=0
$$

where by integrating twice we obtain

$$
(b+c-m) \varphi+\frac{a}{n+1} \varphi^{n+1}+\varphi^{\prime \prime}=0
$$

substituting (4) into (20) gives

$$
\begin{aligned}
& (b+c-m) \lambda \sin ^{\beta}(\mu \xi)+\frac{a}{n+1} \lambda^{(n+1)} \sin ^{(n+1) \beta}(\mu \xi) \\
& \left.-\mu^{2} \beta^{2} \lambda \sin ^{\beta}(\mu \xi)+\mu^{2} \lambda \beta(\beta-1) \sin ^{\beta-2}(\mu \xi)\right)=0
\end{aligned}
$$

Equating the exponents and the coefficients of each pair of the sine functions we find the following system of algebraic equations:

$$
\begin{aligned}
& (\beta-1) \neq 0, \\
& \beta-2=(n+1) \beta, \\
& (b+c-m) \lambda-\mu^{2} \beta^{2} \lambda=0, \\
& \lambda \mu^{2} \beta(\beta-1)+\frac{a}{n+1} \lambda^{(n+1)}=0
\end{aligned}
$$

solving the system (22) yields

$$
\begin{aligned}
& \beta=-\frac{2}{n}, \mu=\frac{1}{2} \sqrt{b+c-m}, \\
& \lambda=\left[\frac{(n+2)(n+1)(m-b-c)}{2 a}\right]^{\frac{1}{n}},
\end{aligned}
$$

where $m$ is a free parameter. Hence, for $b+c>m$, the following periodic solutions

$$
\begin{aligned}
& u(\xi)= \\
& \left\{\frac{(n+2)(n+1)(m-b-c)}{2 a} \csc ^{2}\left[\frac{\sqrt{b+c-m}}{2}(\xi)\right]\right\}^{\frac{1}{n}},
\end{aligned}
$$

where $0<\frac{\sqrt{b+c-m}}{2}(\xi)<\pi$, and 


$$
u(\xi)=\left\{\frac{(n+2)(n+1)(m-b-c)}{2 a} \sec ^{2}\left[\frac{\sqrt{b+c-m}}{2}(\xi)\right]\right\}^{\frac{1}{n}}
$$

where $\left|\frac{\sqrt{b+c-m}}{2}(\xi)\right|<\frac{\pi}{2}$.

However, for $b+c<m$, the following periodic solutions

$$
\begin{aligned}
& u(\xi)=\left\{\frac{(n+2)(n+1)(b+c-m)}{2 a} \operatorname{csch}^{2}\left[\frac{\sqrt{m-b-c}}{2}(\xi)\right]\right\}^{\frac{1}{n}}, \\
& u(\xi)=\left\{\frac{(n+2)(n+1)(m-b-c)}{2 a} \operatorname{sech}^{2}\left[\frac{\sqrt{b+c-m}}{2}(\xi)\right]\right\}^{\frac{1}{n}} .
\end{aligned}
$$

\section{Conclusions}

In this paper, by using the sine-cosine method, we obtained some new explicit formulas of solutions for the generalized $(1+2)$-dimensional and the generalized $(1+3)$ dimensional KP equations. Those solutions were similar to the solutions obtained in other paper. The study reveals the power of the method.

\section{REFERENCES}

[1] A.M. Wazwaz. (2005) The tanh method: solitons and periodic solutions for the Dodd-Bullough-Tzikhailov and the Tzitzeica-Dodd-Bullough equations, Chaos, Solitons and Fractals, 25, 55-63

[2] A.M. Wazwaz. (2005) The tanh method and the sine-cosine method for solving the KP-MEW equation, Int. J. Comput. Math., 82 (2) , 235-246

[3] M.A. Helal, M.S. Mehanna. (2007) The tanh method and Adomian decomposition method for solving the foam drainage equation, Appl. Math. Comput., 190 (1), 599-609

[4] A.M. Wazwaz. (2007) The extended tanh method for new solitons solutions for many forms of the fifth-order $\mathrm{KdV}$ equations, Appl. Math. Comput., 184 (2) 1002-1014

[5] A.M. Wazwaz. (2007) The extended tanh method for abundant solitary wave solutions of nonlinear wave equations, Appl. Math. Comput., 187, 1131-1142

[6] A.M. Wazwaz. (2007) New solitons and kinks solutions to the Sharma-Tasso-Olver equation, Appl. Math. Comput., 188 (2), $1205-1213$

[7] A.M. Wazwaz. (2004) A sine-cosine method for handling nonlinear wave equations, Math. Comput. Model., 40 499-508

[8] A.M. Wazwaz. (2004) The sine-cosine method for obtaining solutions with compact and noncompact structures, Appl. Math. Comput. , 159 (2), 559-576

[9] E. Yusufoglu, A. Bekir. (2006) Solitons and periodic solutions of coupled nonlinear evolution equations by using sine-cosine method, Int. J. Comput. Math.., 83(12) ,915-924

[10] F. Tascan, A. Bekir. (2009) Analytic solutions of the $(2+$ 1)-dimensional nonlinear evolution equations using the sine-cosine method, Appl. Math. Comput., 215, 3134-3139

[11] Z. Xiqiang, W. Limin, S. Weijun. (2006) The repeated homogeneous balance method and its applications to nonlinear partial differential equations, Chaos, Solitons and Fractals, 28(2), 448-453

[12] S.J. Liao, Beyond Perturbation: Introduction to the homotopy analysis method, Chapman \& Hall/CRC Press, Boca Raton, 2003

[13] S.J. Liao. (2004) On the homotopy analysis method for nonlinear problems, Appl. Math. Comput.., 147, 499-513

[14] S.J. Liao. (2005) A new branch of solutions of boundary-layer flows over an impermeable stretched plate, Int. J. Heat Mass Transfer, 48, 2529-2539

[15] S.J. Liao. (2009) A general approach to get series solution of non-similarity boundary-layer flows, Commun. Nonlinear Sci. Numer. Simul., 14(5), 2144-2159

[16] M.T. Darvishi, F. Khani. (2009) A series solution of the foam drainage equation, Comput. Math. Appl., 58, 360-368

[17] A. Aziz, F. Khani, M.T. Darvishi. (2010) Homotopy analysis method for variable thermal conductivity heat flux gage with edge contact resistance, Zeitschrift fuer Naturforschung A, $65 \mathrm{a}(10), 771-776$

[18] F. Khani, M.T. Darvishi, R.S.R. Gorla. (2011) Analytical investigation for cooling turbine disks with a non-Newtonian viscoelastic fluid, Comput. Math. Appl., 61(7), 1728-1738

[19] E. Fan, Z. Jian. (2002) Applications of the Jacobi elliptic function method to special-type nonlinear equations, Phys. Lett. A., 305 (6), 383-392

[20] M.T. Darvishi, Maliheh Najafi, Mohammad Najafi. (2010) Exact three-wave solutions for high nonlinear form of Benjamin-Bona-Mahony-Burgers equations, International Journal of Mathematical and Computer Sciences, 6(3), 127-131

[21] M.T. Darvishi, Mohammad Najafi. (2012) Some exact solutions of the (2+1)-dimensional breaking soliton equation using the three-wave method, World Academy of Science, Engineering and Technology, 87, 31-34

[22] M.T. Darvishi, Maliheh Najafi, Mohammad Najafi. (2010) New exact solutions for the $(3+1)$-dimensional breaking soliton equation, International Journal of Information and Mathematical Sciences, 6(2), 134-137

[23] M.T. Darvishi, Maliheh Najafi, Mohammad Najafi. (2010) New application of EHTA for the generalized $(2+1)$ -dimensional nonlinear evolution equations, International Journal of Mathematical and Computer Sciences, 6(3), $132-138$

[24] M.T. Darvishi, Mohammad Najafi. (2011) A modification of extended homoclinic test approach to solve the $(3+1)$ dimensional potential-YTSF equation, Chin. Phys. Lett., 28(4), art. no. 040202

[25] M.T. Darvishi, Mohammad Najafi. (2012) Some complexiton 
type solutions of the (3+1)-dimensional Jimbo-Miwa equation, World Academy of Science, Engineering and Technology, 87, 42-44

[26] M.T. Darvishi, Maliheh Najafi, Mohammad Najafi. (2012) Traveling wave solutions for the (3+1)-dimensional breaking soliton equation by $\left(\mathrm{G}^{\prime} / \mathrm{G}\right)$-expansion method and modified F-expansion method, World Academy of Science, Engineering and Technology, 88, 37-42

[27] J.H. He, M.A. Abdou. (2007) New periodic solutions for nonlinear evolution equations using Exp-function method, Chaos, Solitons and Fractals, 34, 1421-1429

[28] F. Khani, S. Hamedi-Nezhad, M.T. Darvishi, S.-W. Ryu (2009) New solitary wave and periodic solutions of the foam drainage equation using the Exp-function method, Nonlin. Anal.: Real World Appl., 10, 1904-1911
[29] B.C. Shin, M.T. Darvishi, A. Barati. (2009) Some exact and new solutions of the Nizhnik-Novikov-Vesselov equation using the Exp-function method, Comput. Math. Appl., $58(11 / 12), 2147-2151$

[30] X.H. Wu, J.H. He. (2008) Exp-function method and its application to nonlinear equations, Chaos, Solitons and Fractals, 38(3), 903-910

[31] A. Biswas. (2010) A Topological 1-soliton solution of Kadomtsev-Petviashvili equation with power law nonlinearity, Appl. Math. Comput., 217, 1771-1773

[32] A. R. Adem, C. M. Khalique, A. Biswas. (2011) Solutions of Kadomtsev-Petviashvili equation with power law nonlinearity in $(1+3)$ dimensions, Math. Meth. Appl. Sci., 34 $532-543$ 